\title{
Pacific
}

Journal of

Mathematics

\section{NORMAL ENVELOPING ALGEBRAS}

AlEXANDRE N. GRISHKov, Marina RASSKaZOVA AND SALVATORE SicILIANO 


\title{
NORMAL ENVELOPING ALGEBRAS
}

\author{
Alexandre N. GrishKov, Marina Rasskazova \\ AND SALVATORE SiCILIANO
}

\begin{abstract}
A full characterization is given of ordinary and restricted enveloping algebras which are normal with respect to the principal involution.
\end{abstract}

\section{Introduction}

Let $A$ be an algebra with involution $*$ over a field $\mathbb{F}$. We recall that $A$ is said to be normal if $x x^{*}=x^{*} x$ for every $x \in A$. Over the decades, normal algebras with involutions have been extensively investigated on their own; see, for example, [Beidar et al. 1981; Bovdi et al. 1985; Bovdi 1990; 1997; Bovdi and Siciliano 2007; Brešar and Vukman 1989; Herstein 1976; Knus et al. 1998; Lim 1977; 1979; Maxwell 1972]. Moreover, they have several applications in linear algebra and functional analysis; see, for example, [Berberian 1959; Fuglede 1950; Maxwell 1972; Mosić and Djordjević 2009; Putnam 1951; Yood 1974]. It is well-known that any normal algebra with involution satisfies the standard polynomial identity of degree 4 [Herstein 1976, Section 5]. Moreover, Maxwell [1972] determined the structure of a normal simple algebra of matrices with entries in a field with involution. He also proved that a division algebra $D$ with involution is normal if and only if $D$ is either a field or a generalized quaternion algebra over its center. Furthermore, a characterization of group algebras which are normal under the standard involution was established by Bovdi, Gudivok, and Semirot [Bovdi et al. 1985]. Subsequently, such a result has been extended to twisted group algebras [Bovdi 1990; 1997] and to group algebras under a Novikov involution [Bovdi and Siciliano 2007].

On the other hand, it seems that the rather natural problems of characterizing ordinary and restricted enveloping algebras which are normal under their canonical involutions have not been settled yet. The present paper is just devoted to answering these questions.

For an arbitrary Lie algebra $L$ we denote by $U(L)$ the universal enveloping algebra of $L$. Moreover, if $L$ is restricted with a $p$-map [ $p$ ] over a field $\mathbb{F}$ of

The first author was supported by FAPESP and CNPq (Brazil) and grant RFFI-10.01.00383a (Russia).

MSC2010: 16S30, 16W10, 17B50.

Keywords: restricted Lie algebra, enveloping algebra, normal ring, principal involution. 
characteristic $p>0$, then we denote by $u(L)$ the restricted enveloping algebra of $L$. We consider $U(L)$ and $u(L)$ with the principal involution $*$, namely, the unique $\mathbb{F}$-antiautomorphism such that $x^{*}=-x$ for every $x$ in $L$; see [Bourbaki 2007, Section 2] or [Dixmier 1974, Section 2]. Note that $*$ is just the antipode of the $\mathbb{F}$-Hopf algebras $U(L)$ or $u(L)$.

We use the symbols $Z(L)$ and $L^{\prime}$ for the center of $L$ and the derived subalgebra of $L$, respectively. If $S \subseteq L$, we denote by $\langle S\rangle_{\mathbb{F}}$ the $\mathbb{F}$-vector space generated by $S$. Also, if $L$ is restricted, $\langle S\rangle_{p}$ denotes the restricted subalgebra generated by $S$, and we put $S^{[p]}=\left\{x^{[p]} \mid x \in S\right\}$. In our first main result we completely settle the restricted case:

Theorem 1.1. Let $L$ be a restricted Lie algebra over a field $\mathbb{F}$ of characteristic $p>0$. Then $u(L)$ is normal if and only if either $L$ is abelian or $p=2, L$ is nilpotent of class 2, and one of the following conditions holds:

(i) L contains an abelian restricted ideal I of codimension 1 .

(ii) $\operatorname{dim}_{\mathbb{F}} L / Z(L)=3$.

(iii) $\operatorname{dim}_{\mathbb{F}} L^{\prime}=1$ and $\left(L^{\prime}\right)^{[2]}=0$.

(iv) $L=\left\langle x, x_{1}, x_{2}, x_{3}\right\rangle_{p}+Z(L)$ with

$$
\begin{aligned}
& {\left[x_{1}, x_{2}\right]=\xi\left[x, x_{3}\right],} \\
& {\left[x_{1}, x_{3}\right]=\mu\left[x, x_{2}\right],} \\
& {\left[x_{2}, x_{3}\right]=\lambda\left[x, x_{1}\right],}
\end{aligned}
$$

and

$$
\lambda\left[x, x_{1}\right]^{[2]}+\mu\left[x, x_{2}\right]^{[2]}+\xi\left[x, x_{3}\right]^{[2]}=0
$$

for some $\lambda, \mu, \xi \in \mathbb{F}$.

Afterwards we apply Theorem 1.1 in order to solve the ordinary case:

Theorem 1.2. Let $L$ be a Lie algebra over an arbitrary field $\mathbb{F}$. Then $U(L)$ is normal if and only if either $L$ is abelian or $p=2, L$ is nilpotent of class 2 , and one of the following conditions holds:

(i) L contains an abelian ideal of codimension 1.

(ii) $\operatorname{dim}_{\mathbb{F}} L / Z(L)=3$.

\section{Proofs}

For any associative algebra $A$, we shall consider the Lie bracket on $A$ defined by $[a, b]:=a b-b a \in A, a, b \in A$. The symbol $Z(A)$ will denote the center of $A$. Moreover, for a subset $S$ of a Lie algebra $L$ we shall denote by $C_{L}(S)$ the centralizer of $S$ in $L$. 
It is easy to verify that a normal algebra with involution satisfies the $*$-polynomial identity $[x, y]=\left[x^{*}, y^{*}\right]$. The converse is also true in characteristic different from 2, but in general it fails without such an assumption [Lim 1977]. However, for restricted Lie algebras we have the following:

Lemma 2.1. Let $L$ be a restricted Lie algebra over a field $\mathbb{F}$ of characteristic 2 such that $[x, y]=\left[x^{*}, y^{*}\right]$ for every $x, y \in u(L)$. Then $L$ is nilpotent of class at most 2 and $u(L)$ is normal.

Proof. For every $a, b, c \in L$, we have

$$
0=[a b, c]+\left[(a b)^{*}, c^{*}\right]=[[a, b], c] .
$$

Hence $L$ is nilpotent of class at most 2 .

Let $\left(e_{i}\right)_{i \in I}$ be an ordered $\mathbb{F}$-basis of $L$. Then every element $u$ of $u(L)$ is an $\mathbb{F}$ linear combination of elements $e_{i_{1}} \cdots e_{i_{m}}$, where $m \geq 0$ and the indices $i_{1}<\cdots<i_{m}$ are in $I$. As $L$ is nilpotent of class at most 2, for every $z \in L$ we have $z^{[2]} \in Z(L)$, and then

$$
\left[e_{i_{1}} \cdots e_{i_{m}},\left(e_{i_{1}} \cdots e_{i_{m}}\right)^{*}\right]=0
$$

Moreover, by hypothesis we clearly have $\left[x, y^{*}\right]=\left[x^{*}, y\right]$ for every $x, y \in u(L)$. We conclude that $\left[u, u^{*}\right]=0$, so that $u(L)$ is normal.

Lemma 2.2. Let $L$ be a restricted Lie algebra over a field $\mathbb{F}$ of characteristic $p>0$ such that $u(L)$ is normal. Then either $L$ is abelian, or $p=2$ and $L$ is nilpotent of class 2.

Proof. As $u(L)$ satisfies the $*$-polynomial identity $[x, y]=\left[x^{*}, y^{*}\right]$, if $p=2$, Lemma 2.1 assures that $L$ is nilpotent of class at most 2. Now suppose $p>2$. For every $x, y \in L$, we have

$$
0=\left[x^{2}+y,\left(x^{2}+y\right)^{*}\right]=-4 x[x, y]+2[x,[x, y]] .
$$

Since $p>2$, in view of the Poincaré-Birkhoff-Witt (PBW) theorem for restricted Lie algebras [Strade and Farnsteiner 1988, Section 2, Theorem 5.1], the previous relation is possible only when $[x, y]=0$, so that $L$ is abelian. This yields the claim.

Let $L$ be a restricted Lie algebra over a field of characteristic 2. For every $a, b, c, d \in L$, we put

$$
\Theta(a, b, c, d):=[a, b][c, d]+[a, c][b, d]+[a, d][b, c] \in u(L) .
$$

The following result will be extremely useful in the sequel. 
Lemma 2.3. Let $L$ be a restricted Lie algebra over a field $\mathbb{F}$ of characteristic 2 , and suppose $L$ to be nilpotent of class 2 . Then $u(L)$ is normal if and only if $\Theta(a, b, c, d)=0$ for all $a, b, c, d \in L$.

Proof. If $u(L)$ is normal, for all $a, b, c, d \in L$ we have

$$
\Theta(a, b, c, d)=[a, b c d]+[a, d c b]=[a, b c d]+\left[a,(b c d)^{*}\right]=0 .
$$

Conversely, assume that $\Theta(a, b, c, d)=0$ for all $a, b, c, d \in L$. Let $\left(e_{j}\right)_{j \in J}$ be an ordered $\mathbb{F}$-basis of $L$ containing an $\mathbb{F}$-basis of $Z(L)$. Since $u(L)$ is a free $u(Z(L))$ module, there exists a unique homomorphism of $u(Z(L))$-modules

$$
\phi: u(L) \rightarrow u(L)
$$

which vanishes on 1 and $L$, and such that for every $n>1$ and $j_{1}<\ldots<j_{n}$, one has

$$
\phi\left(e_{j_{1}} \cdots e_{j_{n}}\right)=\sum_{1 \leq h<k \leq n} e_{j_{1}} \cdots \hat{e}_{j_{h}} \cdots \hat{e}_{j_{k}} \cdots e_{j_{n}}\left[e_{j_{h}}, e_{j_{k}}\right],
$$

where the symbol $\hat{e}_{i_{h}}$ indicates that $e_{i_{h}}$ is to be omitted.

We claim that

$$
\operatorname{Im}(\phi) \subseteq Z(u(L))
$$

For this purpose it is enough to prove that $\left[x, \phi\left(e_{j_{1}} \cdots e_{j_{n}}\right)\right]=0$ for every $x \in L$, $n>1$, and $j_{1}, \ldots, j_{n} \in J$ with $j_{1}<\ldots<j_{n}$. Indeed, by the hypothesis we have

$$
\begin{aligned}
{\left[x, \phi\left(e_{j_{1}} \cdots e_{j_{n}}\right)\right] } & =\left[x, \sum_{1 \leq h<k \leq n} e_{j_{1}} \cdots \hat{e}_{j_{h}} \cdots \hat{e}_{j_{k}} \cdots e_{j_{n}}\left[e_{j_{h}}, e_{j_{k}}\right]\right] \\
& =\sum_{1 \leq h<k \leq n} \sum_{\substack{1 \leq s \leq n \\
s \neq h, k}} e_{j_{1}} \cdots \hat{e}_{j_{h}} \cdots \hat{e}_{j_{s}} \cdots \hat{e}_{j_{k}} \cdots e_{j_{n}}\left[e_{j_{h}}, e_{j_{k}}\right]\left[x, e_{j_{s}}\right] \\
& =\sum_{1 \leq h<k<s \leq n} e_{j_{1}} \cdots \hat{e}_{j_{h}} \cdots \hat{e}_{j_{k}} \cdots \hat{e}_{j_{s}} \cdots e_{j_{n}}\left(\left[e_{j_{h}}, e_{i_{k}}\right]\left[x, e_{j_{s}}\right]\right. \\
& \left.+\left[e_{j_{h}}, e_{i_{s}}\right]\left[x, e_{j_{k}}\right]+\left[e_{j_{k}}, e_{i_{s}}\right]\left[x, e_{j_{h}}\right]\right)=0,
\end{aligned}
$$

yielding the claim.

Now we shall prove that

$$
a=a^{*}+\phi(a)
$$

for every $a \in u(L)$. For this purpose it is enough to show that for all $n \geq 0$ and $j_{1}, \ldots, j_{n} \in J$ with $j_{1}<\ldots<j_{n}$, one has

$$
e_{j_{1}} \cdots e_{j_{n}}=e_{j_{n}} \cdots e_{j_{1}}+\phi\left(e_{j_{1}} \cdots e_{j_{n}}\right)
$$


Let us proceed by induction on $n$. By the proved claim and the inductive assumption, we have, for $n>0$,

$$
\begin{aligned}
& e_{j_{1}} \cdots e_{j_{n}} \\
& \quad=\left(e_{j_{n-1}} \cdots e_{j_{1}}\right) e_{j_{n}}+\phi\left(e_{j_{1}} \cdots e_{j_{n-1}}\right) e_{j_{n}} \\
& =e_{j_{n}} e_{j_{n-1}} \cdots e_{j_{1}}+\left[e_{j_{n-1}} \cdots e_{j_{1}}, e_{j_{n}}\right]+\phi\left(e_{j_{1}} \cdots e_{j_{n-1}}\right) e_{j_{n}} \\
& =e_{j_{n}} e_{j_{n-1}} \cdots e_{j_{1}}+\left[e_{j_{1}} \cdots e_{j_{n-1}}, e_{j_{n}}\right]+\left[\phi\left(e_{j_{1}} \cdots e_{j_{n-1}}\right), e_{j_{n}}\right]+\phi\left(e_{j_{1}} \cdots e_{j_{n-1}}\right) e_{j_{n}} \\
& =e_{j_{n}} \cdots e_{j_{1}}+\phi\left(e_{j_{1}} \cdots e_{j_{n}}\right),
\end{aligned}
$$

completing the inductive step.

Finally, by applying the properties proved above, for all $a, b \in u(L)$, we have

$$
[a, b]=\left[a^{*}+\phi(a), b^{*}+\phi(b)\right]=\left[a^{*}, b^{*}\right] .
$$

Hence $u(L)$ is normal by Lemma 2.1, as required.

Remark 2.4. Since $\Theta$ is an alternating $\mathbb{F}$-multilinear function, by Lemma 2.3 it is clear that in order to conclude that $u(L)$ is normal, it suffices to check that $\Theta(a, b, c, d)=0$ for all pairwise distinct noncentral elements $a, b, c, d$ in a fixed $\mathbb{F}$-basis of $L$.

We are now in position to prove Theorem 1.1:

Proof of Theorem 1.1. Assume that $u(L)$ is normal and $L$ is not abelian. Then, by Lemma 2.3, we know that $\mathbb{F}$ has characteristic 2 and $L$ is nilpotent of class 2 . Let us proceed with a case-by-case analysis.

Case 1. $\max \left\{\operatorname{dim}_{\mathbb{F}}[L, x] \mid x \in L\right\}=1$. Let $x_{1}$ and $y_{1}$ be two noncommuting element of $L$ and put $z_{1}:=\left[x_{1}, y_{1}\right]$. By assumption we have $\left[L, x_{1}\right]=\left[L, y_{1}\right]=\mathbb{F} z_{1}$ and $L=\mathbb{F} y_{1} \oplus C_{L}\left(x_{1}\right)$. Now, if $C_{L}\left(x_{1}\right)$ is abelian, $L$ satisfies alternative (i) of the statement. Suppose then that there exist $x_{2}, y_{2} \in C_{L}\left(x_{1}\right)$ such that $\left[x_{2}, y_{2}\right]:=z_{2} \neq 0$. From Lemma 2.3 it follows that

$$
z_{1} z_{2}=\Theta\left(x_{1}, y_{1}, x_{2}, y_{2}\right)=0 .
$$

Therefore the PBW theorem for restricted Lie algebras entails that $z_{1}=\lambda z_{2}$ for some $\lambda \in \mathbb{F}$, which shows that $L^{\prime}=\mathbb{F} z_{1}$. Also, as $\lambda \neq 0$, by (1), we have $z_{1}^{[2]}=0$. Thus $\left(L^{\prime}\right)^{[2]}=0$, and alternative (iii) of the statement holds.

Case 2. $\max \left\{\operatorname{dim}_{\mathbb{F}}[L, x] \mid x \in L\right\}=2$. Let $x, x_{1}, x_{2} \in L$ such that $z_{1}:=\left[x, x_{1}\right]$ and $z_{2}:=\left[x, x_{2}\right]$ are $\mathbb{F}$-linearly independent. We clearly have $L=\left\langle x_{1}, x_{2}\right\rangle_{\mathbb{F}} \oplus C_{L}(x)$. Furthermore, by Lemma 2.3, we have, for all $y_{1}, y_{2} \in C_{L}(x)$,

$$
0=\Theta\left(x, x_{1}, y_{1}, y_{2}\right)=z_{1}\left[y_{1}, y_{2}\right] \quad \text { and } \quad 0=\Theta\left(x, x_{2}, y_{1}, y_{2}\right)=z_{2}\left[y_{1}, y_{2}\right] \text {. }
$$


Since $z_{1}$ and $z_{2}$ are $\mathbb{F}$-linearly independent, the PBW theorem forces $\left[y_{1}, y_{2}\right]=0$. Hence $C_{L}(x)$ is abelian. Again by Lemma 2.3, for every $y \in C_{L}(x)$, we have

$$
0=\Theta\left(x, x_{1}, x_{2}, y\right)=z_{1}\left[x_{2}, y\right]+z_{2}\left[x_{1}, y\right] .
$$

At this stage, a straightforward application of the PBW theorem yields

$$
\left[x_{1}, y\right]=\lambda_{11}(y) z_{1}+\lambda_{12}(y) z_{2} \quad \text { and } \quad\left[x_{2}, y\right]=\lambda_{21}(y) z_{1}+\lambda_{22}(y) z_{2}
$$

for some $\lambda_{11}(y), \lambda_{12}(y), \lambda_{21}(y), \lambda_{22}(y) \in \mathbb{F}$. From (2) it follows that

$$
\left(\lambda_{11}(y)+\lambda_{22}(y)\right) z_{1} z_{2}=\lambda_{21}(y) z_{1}^{2}+\lambda_{12}(y) z_{2}^{2} \in L,
$$

and, again by the PBW theorem, the preceding relation is possible only when $\lambda_{11}(y)=\lambda_{22}(y):=\lambda(y)$. With the notation just introduced, we consider the following subcases.

Subcase 2.1. For every $u \in C_{L}(x)$, one has $\lambda_{12}(u)=\lambda_{21}(u)=0$. Let $y \in C_{L}(x)$ and put $\bar{y}:=\lambda(y) x+y$. Then we have $[\bar{y}, x]=\left[\bar{y}, x_{1}\right]=\left[\bar{y}, x_{2}\right]=0$. As $C_{L}(x)$ is abelian, it follows that $\bar{y} \in Z(L)$ and then $C_{L}(x)=\mathbb{F} x \oplus Z(L)$. Thus $\operatorname{dim}_{\mathbb{F}} L / Z(L)=3$, and alternative (ii) of the statement holds.

Subcase 2.2. There exists $u \in C_{L}(x)$ such that $\lambda_{12}(u) \neq 0$ and $\lambda_{21}(u)=0$. By replacing $u$ by $\lambda_{12}^{-1}(u) u$, we can suppose that $\lambda_{12}(u)=1$. Put $y:=\lambda(u) x+u$. Then we have

$$
\left[x_{1}, y\right]=z_{2} \quad \text { and } \quad\left[x_{2}, y\right]=0 .
$$

Let $y_{1} \in C_{L}(x)$. Since $C_{L}(x)$ is abelian, by Lemma 2.3 we have

$$
0=\Theta\left(x_{1}, x_{2}, y, y_{1}\right)=z_{2}\left[x_{2}, y_{1}\right]=z_{2}\left(\lambda_{21}\left(y_{1}\right) z_{1}+\lambda\left(y_{1}\right) z_{2}\right) .
$$

Consequently, as $z_{1}$ and $z_{2}$ are $\mathbb{F}$-linearly independent, the PBW theorem forces $\lambda_{21}\left(y_{1}\right)=0$. Also, from relation (3) (applied for $y_{1}=x$ ), we infer that $z_{2}^{[2]}=0$. Now put $\bar{y}_{1}:=\lambda\left(y_{1}\right) x+\lambda_{12}\left(y_{1}\right) y+y_{1}$. Then $\bar{y}_{1} \in Z(L)$, and $C_{L}(x)=\mathbb{F} x \oplus \mathbb{F} y \oplus Z(L)$. We conclude that $L=\left\langle x, x_{1}, x_{2}, y\right\rangle_{p}+Z(L)$, and it is clear that $L$ is a restricted Lie algebra satisfying alternative (iv) of the statement.

Subcase 2.3. There exists $u \in C_{L}(x)$ such that $\lambda_{12}(u)=0$ and $\lambda_{21}(u) \neq 0$. This is analogous to Subcase 2.2.

Subcase 2.4. There exists $u \in C_{L}(x)$ such that $\lambda_{12}(u) \neq 0$ and $\lambda_{21}(u) \neq 0$. By replacing $u$ by $\lambda_{12}^{-1}(u) u$, we can suppose that $\lambda_{12}(u)=1$. Put $y:=\lambda(u) x+u$. Then we have

$$
\left[x_{1}, y\right]=z_{2} \quad \text { and } \quad\left[x_{2}, y\right]=\lambda_{21}(u) z_{1} .
$$

Moreover, Lemma 2.3 yields

$$
0=\Theta\left(x, x_{1}, x_{2}, y\right)=\lambda_{21}(u) z_{1}^{2}+z_{2}^{2} .
$$


Let $y_{1} \in C_{L}(x)$ and put $\bar{y}_{1}:=\lambda\left(y_{1}\right) x+y_{1}$. As $C_{L}(x)$ is abelian, Lemma 2.3 yields $0=\Theta\left(x_{1}, x_{2}, y, \bar{y}_{1}\right)=z_{2}\left[x_{2}, \bar{y}_{1}\right]+\lambda_{21}(u) z_{1}\left[x_{1}, \bar{y}_{1}\right]=\left(\lambda_{21}\left(\bar{y}_{1}\right)+\lambda_{21}(u) \lambda_{12}\left(\bar{y}_{1}\right)\right) z_{1} z_{2}$, so that $\lambda_{21}\left(\bar{y}_{1}\right)=\lambda_{21}(u) \lambda_{12}\left(\bar{y}_{1}\right)$. Put $\hat{y}_{1}:=\bar{y}_{1}+\lambda_{12}\left(\bar{y}_{1}\right) y$. Then we have $\left[x_{1}, \hat{y}_{1}\right]=0$. Now, if for some $y_{1} \in C_{L}(x)$ one has $\left[x_{2}, \hat{y}_{1}\right]=\lambda_{21}\left(\hat{y}_{1}\right) z_{1} \neq 0$ then we can replace $y$ by $\hat{y}_{1}$ and conclude by Subcase 2.3 that alternative (iv) holds. On the other hand, if $\left[x_{2}, \hat{y}_{1}\right]=0$ for every $y_{1} \in C_{L}(x)$ then $L=\left\langle x, x_{1}, x_{2}, y\right\rangle_{p}+Z(L)$, and it is clear that, also in this case, $L$ is a restricted Lie algebra satisfying alternative (iv).

Case 3. $\max \left\{\operatorname{dim}_{\mathbb{F}}[L, x] \mid x \in L\right\}=3$. Let $x, u_{1}, u_{2}, u_{3} \in L$ such that $z_{1}:=\left[x, u_{1}\right]$, $z_{2}:=\left[x, u_{2}\right]$, and $z_{3}:=\left[x, u_{3}\right]$ are $\mathbb{F}$-linearly independent. We clearly have $L=$ $\left\langle u_{1}, u_{2}, u_{3}\right\rangle_{\mathbb{F}} \oplus C_{L}(x)$, and one can show that $C_{L}(x)$ is abelian in the same way as in Case 2. Moreover, in view of Lemma 2.3, we have

$$
0=\Theta\left(x, u_{1}, u_{2}, u_{3}\right)=z_{1}\left[u_{2}, u_{3}\right]+z_{2}\left[u_{1}, u_{3}\right]+z_{3}\left[u_{1}, u_{2}\right] .
$$

Thus, for every $1 \leq i<j \leq 3$, by the PBW theorem, we see that

$$
\left[u_{i}, u_{j}\right]=\sum_{k=1}^{3} \alpha_{i j}^{(k)} z_{k},
$$

where $\alpha_{i j}^{(k)} \in \mathbb{F}, k=1,2,3$. By (4) and (5), another application of the PBW theorem yields

$$
\alpha_{12}^{(1)}=\alpha_{23}^{(3)}, \quad \alpha_{12}^{(2)}=\alpha_{13}^{(3)}, \quad \alpha_{13}^{(1)}=\alpha_{23}^{(2)} .
$$

Put

$$
x_{1}:=u_{1}+\alpha_{12}^{(2)} x, \quad x_{2}:=u_{2}+\alpha_{12}^{(1)} x, \quad x_{3}:=u_{3}+\alpha_{13}^{(1)} x,
$$

and, moreover, $\alpha_{23}^{(1)}:=\lambda, \alpha_{13}^{(2)}:=\mu$, and $\alpha_{12}^{(3)}:=\xi$. Then we have

$$
\left[x_{1}, x_{2}\right]=\xi z_{3}, \quad\left[x_{1}, x_{3}\right]=\mu z_{2}, \quad\left[x_{2}, x_{3}\right]=\lambda z_{1} .
$$

From Lemma 2.3 it follows that

$$
\lambda z_{1}^{[2]}+\mu z_{2}^{[2]}+\xi z_{3}^{[2]}=\Theta\left(x, x_{1}, x_{2}, x_{3}\right)=0 .
$$

Now, let $y \in C_{L}(x)$. By Lemma 2.3 we obtain

$$
\begin{aligned}
& \Theta\left(x, x_{1}, x_{2}, y\right)=z_{1}\left[x_{2}, y\right]+z_{2}\left[x_{1}, y\right]=0, \\
& \Theta\left(x, x_{1}, x_{3}, y\right)=z_{1}\left[x_{3}, y\right]+z_{3}\left[x_{1}, y\right]=0, \\
& \Theta\left(x, x_{2}, x_{3}, y\right)=z_{2}\left[x_{3}, y\right]+z_{3}\left[x_{2}, y\right]=0 .
\end{aligned}
$$

Consequently, by the PBW theorem there exists $\beta \in \mathbb{F}$ such that $\left[x_{i}, y\right]=\beta z_{i}$ for every $i=1,2,3$. Put $\bar{y}:=y+\beta x$. Then $\bar{y} \in Z(L)$ and $C_{L}(x)=\mathbb{F} x \oplus Z(L)$. We conclude that $L=\left\langle x, x_{1}, x_{2}, x_{3}\right\rangle_{p}+Z(L)$, and alternative (iv) is satisfied. 
Case 4. $\max \left\{\operatorname{dim}_{\mathbb{F}}[L, x] \mid x \in L\right\}>3$. Let $S:=\left(u_{i}\right)_{i \in I}$ be a subset of $L$ such that the elements $z_{i}:=\left[x, u_{i}\right], i \in I$, are $\mathbb{F}$-linearly independent, and $[S, x]=[L, x]$. We clearly have $L=\langle S\rangle_{\mathbb{F}} \oplus C_{L}(x)$, and one can show that $C_{L}(x)$ is abelian by proceeding in a similar way as in Case 2. Let $i, j \in I, i \neq j$. In view of Lemma 2.3, for every $k \in I \backslash\{i, j\}$, we have

$$
0=\Theta\left(x, u_{i}, u_{j}, u_{k}\right)=z_{i}\left[u_{j}, u_{k}\right]+z_{j}\left[u_{i}, u_{k}\right]+z_{k}\left[u_{i}, u_{j}\right] .
$$

At this stage, by arguing as in the first case of Case 3 , we have that $\left[u_{i}, u_{j}\right] \in \mathbb{F} z_{k}$. As $|I|>3$, we conclude that $\left[u_{i}, u_{j}\right]=0$. Finally, let $y \in C_{L}(x)$. By Lemma 2.3, for all pairwise distinct elements $i, j, k$ of $I$, we have

$$
\begin{aligned}
& \Theta\left(x, u_{i}, u_{j}, y\right)=z_{i}\left[u_{j}, y\right]+z_{j}\left[u_{i}, y\right]=0, \\
& \Theta\left(x, u_{i}, u_{k}, y\right)=z_{i}\left[x_{k}, y\right]+z_{k}\left[u_{i}, y\right]=0 .
\end{aligned}
$$

Therefore, an application of the PBW theorem shows that there exists $\beta \in \mathbb{F}$ such that $\left[u_{i}, y\right]=\beta z_{i}$ for every $i \in I$. Put $\bar{y}:=y+\beta x$. Then $\bar{y} \in Z(L)$, so that $C_{L}(x)=\mathbb{F} x \oplus Z(L)$. Therefore, as $L^{[2]} \subseteq Z(L)$, we conclude that $Z(L)+\langle S\rangle_{\mathbb{F}}$ is an abelian restricted ideal of codimension 1 in $L$, and the proof of the necessity part is finished.

Now let us prove sufficiency. The claim is trivial if $L$ is abelian. Then assume that the ground field has characteristic 2 and $L$ is nilpotent of class 2 . If $L$ has an abelian restricted ideal of codimension 1 , it is clear that $\Theta(a, b, c, d)=0$ for any $a, b, c, d \in L$, and so, by Lemma $2.3, u(L)$ is normal. Also, if $\operatorname{dim}_{\mathbb{F}} L / Z(L)=3$ then $u(L)$ is normal by Lemma 2.3 and Remark 2.4. Furthermore, the claim is clear whenever $L^{\prime}=\mathbb{F} z$ for some $0 \neq z \in L$ with $z^{[2]}=0$. Finally suppose that alternative (iv) holds. We can assume that $x, x_{1}, x_{2}$, and $x_{3}$ are $\mathbb{F}$-linearly independent (otherwise alternative (i) or (ii) holds). Extend the set $\left\{x, x_{1}, x_{2}, x_{3}\right\}$ by central elements in order to form an $\mathbb{F}$-basis of $L$. We have

$$
\begin{aligned}
\Theta\left(x, x_{1}, x_{2}, x_{3}\right) & =\left[x, x_{1}\right]\left[x_{2}, x_{3}\right]+\left[x, x_{2}\right]\left[x_{1}, x_{3}\right]+\left[x, x_{3}\right]\left[x_{1}, x_{2}\right] \\
& =\lambda\left[x, x_{1}\right]^{[2]}+\mu\left[x, x_{2}\right]^{[2]}+\xi\left[x, x_{3}\right]^{[2]}=0 .
\end{aligned}
$$

From Lemma 2.3 and Remark 2.4 it follows that $u(L)$ is normal.

Finally, we deal with ordinary universal enveloping algebras of arbitrary Lie algebras. Indeed, we shall prove Theorem 1.2 as a consequence of Theorem 1.1.

Proof of Theorem 1.2. Suppose first that ground field $\mathbb{E}$ has characteristic zero. If $L$ is abelian then $U(L)$ is obviously normal. On the other hand, if $U(L)$ is normal then it satisfies the standard polynomial identity of degree 4 [Herstein 1976, Section 5]. Therefore, in view of a theorem of Latysěv [Bahturin 1987, Section 6.7, 
Theorem 25], $L$ is necessarily abelian. Now suppose $p>0$. Put

$$
\hat{L}:=\sum_{k \geq 0} L^{p^{k}} \subseteq U(L),
$$

where $L^{p^{k}}$ is the $\mathbb{F}$-vector space spanned by the set $\left\{l^{p^{k}} \mid l \in L\right\}$. Then $\hat{L}$ is a restricted Lie algebra with $h^{[p]}=h^{p}$ for all $h \in \hat{L}$. Moreover, by [Strade 2004, Section 1, Corollary 1.1.4], we have $U(L)=u(\hat{L})$, and then Theorem 1.1 applies. Suppose first that $U(L)$ is normal. If $p>2$, Theorem 1.1 forces $\hat{L}$ (and so $L$ ) to be abelian. Now assume that $p=2$ and $L$ is not abelian. Then $\hat{L}$ satisfies one of the alternatives (i)-(iv) in the statement of Theorem 1.1. If $\hat{L}$ contains an abelian restricted ideal of codimension 1 then $L$ contains an abelian ideal of codimension 1 . Likewise, if $\operatorname{dim}_{\mathbb{F}} \hat{L} / Z(\hat{L})=3, \operatorname{dim}_{\mathbb{F}} L / Z(L)=3$. Observe that, as $u(\hat{L})=U(L)$ is a domain, alternative (iii) in the statement of Theorem 1.1 cannot occur. Finally, suppose that $\hat{L}=\left\langle x, x_{1}, x_{2}, x_{3}\right\rangle_{p}+Z(\hat{L})$, where $x, x_{1}, x_{2}$, and $x_{3}$ are elements of $L$ with $\left[x_{1}, x_{2}\right]=\xi\left[x, x_{3}\right],\left[x_{1}, x_{3}\right]=\mu\left[x, x_{2}\right],\left[x_{2}, x_{3}\right]=\lambda\left[x, x_{1}\right]$, and

$$
\lambda\left[x, x_{1}\right]^{[2]}+\mu\left[x, x_{2}\right]^{[2]}+\xi\left[x, x_{3}\right]^{[2]}=0
$$

for some $\lambda, \mu, \xi \in \mathbb{F}$. Now, if $\operatorname{dim}_{\mathbb{F}} L^{\prime}=3$, the PBW theorem for ordinary enveloping algebras forces $\lambda=\mu=\xi=0$. Hence $L$ contains an abelian ideal of codimension 1. If $\operatorname{dim}_{\mathbb{F}} L^{\prime}=2$, we can suppose without loss of generality that $\left[x, x_{1}\right]$ and $\left[x, x_{2}\right]$ are $\mathbb{F}$-linearly independent and $\left[x, x_{3}\right]=\alpha\left[x, x_{1}\right]+\beta\left[x, x_{2}\right]$ for suitable $\alpha, \beta \in \mathbb{F}$. Consequently, we have

$$
\alpha^{2} \xi\left[x, x_{1}\right]^{2}+\beta^{2} \xi\left[x, x_{2}\right]^{2}=\xi\left[x, x_{3}\right]^{2}=\lambda\left[x, x_{1}\right]^{2}+\mu\left[x, x_{2}\right]^{2},
$$

and the PBW theorem gets $\lambda=\alpha^{2} \xi$ and $\mu=\beta^{2} \xi$. Put

$$
y:=\alpha \beta \xi x+\alpha x_{1}+\beta x_{2}+x_{3} .
$$

Then $y \in Z(\hat{L})$ and $\hat{L}=\left\langle x, x_{1}, x_{2}, y\right\rangle_{p}+Z(\hat{L})$. It follows that $\operatorname{dim}_{\mathbb{F}} \hat{L} / Z(\hat{L})=3$ and then $\operatorname{dim}_{\mathbb{F}} L / Z(L)=3$ as well. Finally, if $\operatorname{dim}_{\mathbb{F}} L^{\prime}=1$ then it is easy to see that $L$ contains an abelian ideal of codimension 1 , and the necessity part is proved. Sufficiency easily follows from Theorem 1.1 and the fact that $U(L)=u(\hat{L})$.

\section{Acknowledgement}

We thank W. de Graaf, S. Cicalò, and the referee for useful comments.

\section{References}

[Bahturin 1987] Y. A. Bahturin, Identical relations in Lie algebras, VNU Science Press, Utrecht, 1987. MR 88f:17032 Zbl 0691.17001

[Beidar et al. 1981] C. I. Beidar, A. V. Mikhalev, and C. Salavova, "Generalized identities and semiprime rings with involution”, Math. Z. 178:1 (1981), 37-62. MR 83b:16012 Zbl 0471.16008 
[Berberian 1959] S. K. Berberian, "Note on a theorem of Fuglede and Putnam", Proc. Amer. Math. Soc. 10:2 (1959), 175-182. MR 21 \#6548 Zbl 0092.32004

[Bourbaki 2007] N. Bourbaki, Groupes et algèbres de Lie: Chapitre 1, Springer, Berlin, 2007. MR 42 \#6159 Zbl 1120.17001

[Bovdi 1990] V. A. Bovdi, "Normal twisted group rings", Dokl. Akad. Nauk Ukrain. SSR Ser. A 7 (1990), 6-8. In Russian. MR 91i:20004 Zbl 0718.16020

[Bovdi 1997] V. A. Bovdi, "Structure of normal twisted group rings", Publ. Math. Debrecen 51:3-4 (1997), 279-293. MR 98j:16016 Zbl 0906.16013

[Bovdi and Siciliano 2007] V. A. Bovdi and S. Siciliano, "Normality in group rings", Algebra $i$ Analiz 19:2 (2007), 1-9. In Russian; translated in St. Petersbg. Math. J. 19:2 (2008), 159-165. MR 2008d:16040 Zbl 1200.16036

[Bovdi et al. 1985] A. A. Bovdi, P. M. Gudivok, and M. S. Semirot, "Normal group rings", Ukrain. Mat. Zh. 37:1 (1985), 3-8. In Russian; translated in Ukrain. Math. J. 37:1 (1985), 1-5. MR 86h: 16013 Zbl 0572.16005

[Brešar and Vukman 1989] M. Brešar and J. Vukman, "On some additive mappings in rings with involution”, Aequationes Math. 38:2-3 (1989), 178-185. MR 90j:16076 Zbl 0691.16041

[Dixmier 1974] J. Dixmier, Algèbres enveloppantes, Cahiers Scientifiques 37, Gauthier-Villars, Paris, 1974. Translated as Enveloping algebras, Graduate Studies in Mathematics 11, Amer. Math. Soc., Providence, RI, 1996. MR 58 \#16803a Zbl 0308.17007

[Fuglede 1950] B. Fuglede, "A commutativity theorem for normal operators", Proc. Nat. Acad. Sci. USA 36:1 (1950), 35-40. MR 11,371c Zbl 0035.35804

[Herstein 1976] I. N. Herstein, Rings with involution, University of Chicago Press, Chicago, IL, 1976. MR 56 \#406 Zbl 0343.16011

[Knus et al. 1998] M.-A. Knus, A. Merkurjev, M. Rost, and J.-P. Tignol, The book of involutions, Amer. Math. Soc. Colloq. Publ. 44, Amer. Math. Soc., Providence, RI, 1998. MR 2000a:16031 Zbl 0955.16001

[Lim 1977] T. P. Lim, "Conjugacy of elements in a normal ring", Canad. Math. Bull. 20 (1977), 113-115. MR 56 \#3049 Zbl 0358.16008

[Lim 1979] T. P. Lim, "Some classes of rings with involution satisfying the standard polynomial of degree 4", Pacific J. Math. 85:1 (1979), 125-130. MR 81j:16018 Zbl 0444.16004

[Maxwell 1972] G. Maxwell, "Algebras of normal matrices”, Pacific J. Math. 43:2 (1972), 421-428. MR 47 \#6736 Zbl 0234.15028

[Mosić and Djordjević 2009] D. Mosić and D. S. Djordjević, "Moore-Penrose-invertible normal and Hermitian elements in rings", Linear Algebra Appl. 431:5-7 (2009), 732-745. MR 2011a:16058 Zbl 1186.16046

[Putnam 1951] C. R. Putnam, "On normal operators in Hilbert space”, Amer. J. Math. 73:2 (1951), 357-362. MR 12,717f Zbl 0042.34501

[Strade 2004] H. Strade, Simple Lie algebras over fields of positive characteristic, I: Structure theory, Expositions in Math. 38, De Gruyter, Berlin, 2004. MR 2005c:17025 Zbl 1074.17005

[Strade and Farnsteiner 1988] H. Strade and R. Farnsteiner, Modular Lie algebras and their representations, Pure and Appl. Math. 116, Dekker, New York, 1988. MR 89h:17021 Zbl 0648.17003

[Yood 1974] B. Yood, "Commutativity properties in Banach *-algebras", Pacific J. Math. 53:1 (1974), 307-317. MR 50 \#14247 Zbl 0268.46050

Received June 14, 2011. Revised November 11, 2011. 
ALEXANDRE N. GRISHKOV

INSTITUTO DE MATEMÁticA E EstatísticA

UNIVERSIDAdE DE SÃo PAULO

RUA DO MATÃo, 1010 CitAdE Universitária

CEP 05508-090 SÃo PAUlo

BRAZIL

grishkov@ime.usp.br

MARINA RASSKAZOVA

Omsk Institute of Consumer Service Technology

Ul. PeVzova 13, OMSK 644099

RUSSIA

marras123@gmail.com

SALVATORE SiCILIANO

Dipartimento di Matematica e Fisica "Ennio De Giorgi" UNIVERSITÀ DEL SALENTO

Via PRovinciale LeCCE-ARnesano, 73100-LeCCE

ITALY

salvatore.siciliano@unisalento.it 


\title{
PACIFIC JOURNAL OF MATHEMATICS
}

\author{
http://pacificmath.org \\ Founded in 1951 by \\ E. F. Beckenbach (1906-1982) and F. Wolf (1904-1989)
}

\section{EDITORS}

V. S. Varadarajan (Managing Editor)

Department of Mathematics

University of California

Los Angeles, CA 90095-1555

pacific@math.ucla.edu

Vyjayanthi Chari

Department of Mathematics

University of California

Riverside, CA 92521-0135

chari@math.ucr.edu

\section{Robert Finn}

Department of Mathematics Stanford University

Stanford, CA 94305-2125

finn@math.stanford.edu

Kefeng Liu

Department of Mathematics

University of California

Los Angeles, CA 90095-1555

liu@math.ucla.edu
Darren Long

Department of Mathematics

University of California

Santa Barbara, CA 93106-3080

long@math.ucsb.edu

Jiang-Hua Lu

Department of Mathematics

The University of Hong Kong

Pokfulam Rd., Hong Kong jhlu@maths.hku.hk

Alexander Merkurjev

Department of Mathematics

University of California

Los Angeles, CA 90095-1555

merkurev@math.ucla.edu
Sorin Popa

Department of Mathematics University of California

Los Angeles, CA 90095-1555 popa@math.ucla.edu

Jie Qing

Department of Mathematics

University of California

Santa Cruz, CA 95064

qing@cats.ucsc.edu

Jonathan Rogawski

Department of Mathematics

University of California

Los Angeles, CA 90095-1555

jonr@math.ucla.edu

\section{PRODUCTION}

pacific@math.berkeley.edu

\section{SUPPORTING INSTITUTIONS}

ACADEMIA SINICA, TAIPEI

CALIFORNIA INST. OF TECHNOLOGY INST. DE MATEMÁTICA PURA E APLICADA KEIO UNIVERSITY

MATH. SCIENCES RESEARCH INSTITUTE NEW MEXICO STATE UNIV.

OREGON STATE UNIV.

\author{
STANFORD UNIVERSITY \\ UNIV. OF BRITISH COLUMBIA \\ UNIV. OF CALIFORNIA, BERKELEY \\ UNIV. OF CALIFORNIA, DAVIS \\ UNIV. OF CALIFORNIA, LOS ANGELES \\ UNIV. OF CALIFORNIA, RIVERSIDE \\ UNIV. OF CALIFORNIA, SAN DIEGO \\ UNIV. OF CALIF., SANTA BARBARA
}

\author{
UNIV. OF CALIF., SANTA CRUZ \\ UNIV. OF MONTANA \\ UNIV. OF OREGON \\ UNIV. OF SOUTHERN CALIFORNIA \\ UNIV. OF UTAH \\ UNIV. OF WASHINGTON \\ WASHINGTON STATE UNIVERSITY
}

These supporting institutions contribute to the cost of publication of this Journal, but they are not owners or publishers and have no responsibility for its contents or policies.

See inside back cover or pacificmath.org for submission instructions.

The subscription price for 2012 is US \$420/year for the electronic version, and \$485/year for print and electronic.

Subscriptions, requests for back issues from the last three years and changes of subscribers address should be sent to Pacific Journal of Mathematics, P.O. Box 4163, Berkeley, CA 94704-0163, U.S.A. Prior back issues are obtainable from Periodicals Service Company, 11 Main Street, Germantown, NY 12526-5635. The Pacific Journal of Mathematics is indexed by Mathematical Reviews, Zentralblatt MATH, PASCAL CNRS Index, Referativnyi Zhurnal, Current Mathematical Publications and the Science Citation Index.

The Pacific Journal of Mathematics (ISSN 0030-8730) at the University of California, c/o Department of Mathematics, 969 Evans Hall, Berkeley, CA 94720-3840, is published monthly except July and August. Periodical rate postage paid at Berkeley, CA 94704, and additional mailing offices. POSTMASTER: send address changes to Pacific Journal of Mathematics, P.O. Box 4163, Berkeley, CA 94704-0163.

PJM peer review and production are managed by EditFLOW ${ }^{\mathrm{TM}}$ from Mathematical Sciences Publishers.

PUBLISHED BY PACIFIC JOURNAL OF MATHEMATICS

at the University of California, Berkeley 94720-3840

A NON-PROFIT CORPORATION

Typeset in LATEX

Copyright $(02012$ by Pacific Journal of Mathematics 


\section{PACIFIC JOURNAL OF MATHEMATICS}

Volume $257 \quad$ No. $1 \quad$ May 2012

Energy and volume of vector fields on spherical domains

FABiano G. B. BRito, ANDRÉ O. Gomes and Giovanni S. Nunes

Maps on 3-manifolds given by surgery

BOLDIZSÁR KALMÁR and ANDRÁS I. STIPSICZ

Strong solutions to the compressible liquid crystal system

Yu-Ming ChU, Xian-Gao LiU and XIAO LIU

Presentations for the higher-dimensional Thompson groups $n V$

Johanna HenNig and FranCESCo MatuCCI

Resonant solutions and turning points in an elliptic problem with oscillatory

boundary conditions

ALFONSO CASTRO and ROSA PARDO

Relative measure homology and continuous bounded cohomology of topological pairs

\section{Roberto Frigerio and CRISTINA PAgLiAnTINi}

Normal enveloping algebras

ALEXANDRE N. GrishKov, Marina RASSKazova and SALVATORE SICILIANO

Bounded and unbounded capillary surfaces in a cusp domain

YASUNORI AOKI and DAVID SIEGEL

On orthogonal polynomials with respect to certain discrete Sobolev inner product

Francisco Marcellán, Ramadan Zejnullahu, Bujar Fejzullahu and EDMUNDO HUERTAS

Green versus Lempert functions: A minimal example

PASCAL THOMAS

Differential Harnack inequalities for nonlinear heat equations with potentials under the Ricci flow

JIA-YONG WU

On overtwisted, right-veering open books

PAOLO LISCA

Weakly Krull domains and the composite numerical semigroup ring $D+E\left[\Gamma^{*}\right]$

JUNG WOOK LIM

Arithmeticity of complex hyperbolic triangle groups 